Article

\title{
Generating Functions for New Families of Combinatorial Numbers and Polynomials: Approach to Poisson-Charlier Polynomials and Probability Distribution Function
}

\author{
Irem Kucukoglu ${ }^{1}$, Burcin Simsek ${ }^{2}$ (D) and Yilmaz Simsek ${ }^{3, *(D)}$ \\ 1 Department of Engineering Fundamental Sciences, Faculty of Engineering, Alanya Alaaddin Keykubat \\ University, TR-07425 Antalya, Turkey; irem.kucukoglu@alanya.edu.tr \\ 2 Department of Statistics, University of Pittsburgh, Pittsburgh, PA 15260, USA; bus5@pitt.edu \\ 3 Department of Mathematics, Faculty of Science University of Akdeniz, TR-07058 Antalya, Turkey \\ * Correspondence: ysimsek@akdeniz.edu.tr
}

Received: 14 September 2019; Accepted: 9 October 2019; Published: 11 October 2019

check for updates

\begin{abstract}
The aim of this paper is to construct generating functions for new families of combinatorial numbers and polynomials. By using these generating functions with their functional and differential equations, we not only investigate properties of these new families, but also derive many new identities, relations, derivative formulas, and combinatorial sums with the inclusion of binomials coefficients, falling factorial, the Stirling numbers, the Bell polynomials (i.e., exponential polynomials), the Poisson-Charlier polynomials, combinatorial numbers and polynomials, the Bersntein basis functions, and the probability distribution functions. Furthermore, by applying the $p$-adic integrals and Riemann integral, we obtain some combinatorial sums including the binomial coefficients, falling factorial, the Bernoulli numbers, the Euler numbers, the Stirling numbers, the Bell polynomials (i.e., exponential polynomials), and the Cauchy numbers (or the Bernoulli numbers of the second kind). Finally, we give some remarks and observations on our results related to some probability distributions such as the binomial distribution and the Poisson distribution.
\end{abstract}

Keywords: generating functions; functional equations; partial differential equations; special numbers and polynomials; Bernoulli numbers; Euler numbers; Stirling numbers; Bell polynomials; Cauchy numbers; Poisson-Charlier polynomials; Bernstein basis functions; Daehee numbers and polynomials; combinatorial sums; binomial coefficients; $p$-adic integral; probability distribution

MSC: Primary 05A10; 05A15; 11B73; 11B68; 11B83; Secondary 05A19; 11B37; 11S23; 26C05; 34A99; 35A99; 40C10

\section{Introduction}

In recent years, generating functions and their applications on functional equations and differential equations has gained high attention in various areas. These techniques allow researchers to derive various identities and combinatorial sums that yield important special numbers and polynomials. In fact, the current trend is to combine the $p$-adic integrals with these techniques. In most of fields of mathematics and physics, different applications of generating functions are used as an important tool. For instance, a common research topic in quantum physics is to identify a generating function that could be a solution to a differential equation.

The motivation of this paper is to outline the advantages of techniques associated with generating functions. First, generating functions are presented for new families of combinatorial numbers and polynomials. Second, we derive new identities, relations, and formulas including the Bersntein basis functions, the Stirling numbers, the Bell polynomials (i.e., exponential polynomials), the 
Poisson-Charlier polynomials, the Daehee numbers and polynomials, the probability distribution functions, as well as combinatorial sums including the Bernoulli numbers, the Euler numbers, the Cauchy numbers (or the Bernoulli numbers of the second kind), and combinatorial numbers.

With the followings, we briefly introduce the notations, definitions, relations, and formulas are used throughout this paper:

As usual, let $\mathbb{N}, \mathbb{Z}, \mathbb{N}_{0}, \mathbb{Q}, \mathbb{R}$, and $\mathbb{C}$ denote the set of natural numbers, set of integers, set of nonnegative integers, set of rational numbers, set of real numbers, and set of complex numbers, respectively. Let $\log z$ denote the principal branch of the multi-valued function $\log z$ with the imaginary part $\operatorname{Im}(\log z)$ constrained by the interval $(-\pi, \pi]$. We also assume that:

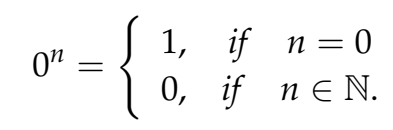

Moreover,

$$
\left(\begin{array}{l}
z \\
v
\end{array}\right)=\frac{z(z-1) \cdots(z-v+1)}{v !}=\frac{(z)_{v}}{v !} \quad(v \in \mathbb{N}, z \in \mathbb{C})
$$

so that,

$$
\left(\begin{array}{l}
z \\
0
\end{array}\right)=(z)_{0}=1
$$

(cf. [1-31]).

The Poisson-Charlier polynomials $C_{n}(x ; a)$, which are members of the family of Sheffer-type sequences, are defined as below:

$$
F_{p c}(t, x ; a)=e^{-t}\left(\frac{t}{a}+1\right)^{x}=\sum_{n=0}^{\infty} C_{n}(x ; a) \frac{t^{n}}{n !}
$$

where,

$$
C_{n}(x ; a)=\sum_{j=0}^{n}(-1)^{n-j}\left(\begin{array}{c}
n \\
j
\end{array}\right) \frac{(x)_{j}}{a^{j}}
$$

(cf. [16], (p. 120, [18]), [24]).

Let $x \in[0,1]$ and let $n$ and $k$ be nonnegative integers. The Bernstein basis functions, $B_{k}^{n}(x)$, are defined by:

$$
B_{k}^{n}(x)=\left(\begin{array}{l}
n \\
k
\end{array}\right) x^{k}(1-x)^{n-k}, \quad(k=0,1, \ldots, n)
$$

so that,

$$
\left(\begin{array}{l}
n \\
k
\end{array}\right)=\frac{n !}{k !(n-k) !}
$$

and its generating function is given by:

$$
F_{B}(t, x ; k)=\frac{(x t)^{k} e^{(1-x) t}}{k !}=\sum_{n=0}^{\infty} B_{k}^{n}(x) \frac{t^{n}}{n !},
$$

where $t \in \mathbb{C}(c f .[1,15,20,26])$.

The Stirling numbers of the first kind, $S_{1}(n, k)$, are defined by the following generating function:

$$
F_{S_{1}}(t ; k)=\frac{(\log (1+t))^{k}}{k !}=\sum_{n=k}^{\infty} S_{1}(n, k) \frac{t^{n}}{n !}, \quad\left(k \in \mathbb{N}_{0}\right)
$$

so that,

$$
(x)_{n}=\sum_{k=0}^{n} S_{1}(n, k) x^{k}
$$


(cf. $[2-4,29,30]$; see also the references cited therein).

The $\lambda$-Stirling numbers of the second kind, $S_{2}(n, k ; \lambda)$, are defined with generating function given below $(c f .[21,30])$ :

$$
F_{S_{2}}(t ; v ; \lambda)=\frac{\left(\lambda e^{t}-1\right)^{v}}{v !}=\sum_{n=0}^{\infty} S_{2}(n, v ; \lambda) \frac{t^{n}}{n !}, \quad\left(v \in \mathbb{N}_{0}\right) .
$$

Notice here that, when $\lambda=1$, this reduces to the Stirling numbers of the second kind, $S_{2}(n, v)$, whose generating function is given below:

$$
F_{S_{2}}(t ; v)=\frac{\left(e^{t}-1\right)^{v}}{v !}=\sum_{n=0}^{\infty} S_{2}(n, v) \frac{t^{n}}{n !}, \quad\left(v \in \mathbb{N}_{0}\right),
$$

namely, $S_{2}(n, v)=S_{2}(n, v ; 1)(c f .[2,5,21,30])$.

The Bell polynomials (i.e., exponential polynomials), $B l_{n}(x)$, is defined by:

$$
B l_{n}(x)=\sum_{v=1}^{n} S_{2}(n, v) x^{v}
$$

so that the generating function for the Bell polynomials is given by:

$$
F_{\text {Bell }}(t, x)=e^{\left(e^{t}-1\right) x}=\sum_{n=0}^{\infty} B l_{n}(x) \frac{t^{n}}{n !}
$$

(cf. $[4,18])$.

The numbers $Y_{n}^{(k)}(\lambda)$ and the polynomials $Y_{n}^{(k)}(x ; \lambda)$ are defined by the following generating functions, respectively:

$$
\mathcal{F}(t, k ; \lambda)=\left(\frac{2}{\lambda(1+\lambda t)-1}\right)^{k}=\sum_{n=0}^{\infty} Y_{n}^{(k)}(\lambda) \frac{t^{n}}{n !},
$$

and,

$$
\mathcal{F}(t, x, k ; \lambda)=\mathcal{F}(t, k ; \lambda)(1+\lambda t)^{x}=\sum_{n=0}^{\infty} Y_{n}^{(k)}(x ; \lambda) \frac{t^{n}}{n !},
$$

where $k \in \mathbb{N}_{0}$ and $\lambda$ is real or complex number (cf. [14]).

Substituting $k=1$ into Equation (11), we have:

$$
Y_{n}(\lambda)=Y_{n}^{(1)}(\lambda)
$$

(cf. [23]).

Substituting $k=1$ and $\lambda=-1$ into Equation (11), we get the following well-known relation between the numbers $Y_{n}(\lambda)$ and the Changhee numbers of the first kind, $C h_{n}$ :

$$
C h_{n}=(-1)^{n+1} Y_{n}(-1) .
$$

Thus we have,

$$
C h_{n}=\frac{(-1)^{n} n !}{n+1}=\sum_{k=0}^{n} S_{1}(n, k) E_{k}
$$

where the Changhee numbers of the first kind, $C h_{n}$ are defined means of the following generating function: 


$$
\frac{2}{t+1}=\sum_{n=0}^{\infty} C h_{n} \frac{t^{n}}{n !}
$$

(cf. [9], see also [7]).

The Daehee polynomials, $D_{n}(x)$, is defined by the following generating functions (cf. [8]):

$$
F_{D}(x, t)=\frac{\log (1+t)}{t}(1+t)^{x}=\sum_{n=0}^{\infty} D_{n}(x) \frac{t^{n}}{n !}
$$

which, for $x=0$, corresponds the generating functions of the Daehee number, $D_{n}=D_{n}(0)$, given by the following explicit formula:

$$
D_{n}=\frac{(-1)^{n} n !}{n+1}
$$

The combinatorial numbers, $y_{1}(n, k ; \lambda)$, are defined by the following generating function:

$$
F_{y_{1}}(t, k ; \lambda)=\frac{1}{k !}\left(\lambda e^{t}+1\right)^{k}=\sum_{n=0}^{\infty} y_{1}(n, k ; \lambda) \frac{t^{n}}{n !}
$$

where $k \in \mathbb{N}_{0}$ and $\lambda \in \mathbb{C}(c f$. [22]).

Use the preceding generating function for the combinatorial numbers, $y_{1}(n, k ; \lambda)$ to compute the following explicit formula:

$$
y_{1}(n, k ; \lambda)=\frac{1}{k !} \sum_{j=0}^{k}\left(\begin{array}{c}
k \\
j
\end{array}\right) \lambda^{j} j^{n}
$$

(cf. [22] (Theorem 1, Equation (9))).

Note that the following equality holds true:

$$
y_{1}(n, k ; \lambda)=\left.\frac{1}{k !} \frac{d^{n}}{d t^{n}}\left(\lambda e^{t}+1\right)^{k}\right|_{t=0}
$$

(cf. [31] (p. 64)).

When $\lambda=1$, if we multiply the numbers $y_{1}(n, k ; \lambda)$ by $k !$, then Equation (18) is reduced to the following combinatorial numbers $(c f .[6,19,22])$ :

$$
B(k, n)=\sum_{j=0}^{k}\left(\begin{array}{l}
k \\
j
\end{array}\right) j^{n}
$$

which satisfies the following differential equation:

$$
B(k, n)=\left.\frac{d^{n}}{d t^{n}}\left(e^{t}+1\right)^{k}\right|_{t=0}
$$

(cf. [6], (Equation (2), p. 2 [22])).

The combinatorial numbers $B(n, k)$ have various kinds of combinatorial applications. For instance, Ross [19] (pp. 18-20, Exercises 10-12) gave the following applications for solutions of exercises 10-12:

From a group of $n$ people, suppose that we want to choose a committee of $k, k \leq n$, one of whom is to be designated as chairperson.

How many different selections are there in which the chairperson and the secretary are the same?

Ross [19] (p. 18, Exercise 12) gave the following answer: $B(1, n)=n 2^{n-1}$.

By using the preceding idea summarized above, the following combinatorial identities are obtained:

$$
\sum_{k=0}^{n}\left(\begin{array}{l}
n \\
k
\end{array}\right) k=n 2^{n-1}
$$




$$
\sum_{k=0}^{n}\left(\begin{array}{l}
n \\
k
\end{array}\right) k^{2}=2^{n-2} n(n+1)
$$

and,

$$
\sum_{k=0}^{n}\left(\begin{array}{l}
n \\
k
\end{array}\right) k^{3}=2^{n-3} n^{2}(n+3)
$$

(cf. (pp. 18-20, Exercises 10-12 [19]), [22,25]). Observe that these numbers are also arised from Equation (20).

Next, we present the outline of the present paper: In Section 2, we construct generating functions for new families of combinatorial numbers and polynomials. By using these generating functions, we not only investigate properties of these new families, but also provide some new identities and relations with the inclusion of the Bersntein basis functions, combinatorial numbers, and the Stirling numbers. In Section 3, we obtain some derivative formulas and recurrence relations for these new families of combinatorial numbers and polynomials by using differential equations that are a result of these generating functions and their partial derivatives. In Section 4, by using functional equations of the generating functions, we derive some formulas and combinatorial sums including binomials coefficients, falling factorial, the Stirling numbers, the Bell polynomials (i.e., exponential polynomials), the Poisson-Charlier polynomials, combinatorial numbers and polynomials, and the Bersntein basis functions. In Section 5, by applying the $p$-adic integrals and Riemann integral to some new formulas derived by the authors of this paper, some combinatorial sums comprising the binomial coefficients, falling factorial, the Bernoulli numbers, the Euler numbers, the Stirling numbers, the Bell polynomials (i.e. exponential polynomials), and the Cauchy numbers (or the Bernoulli numbers of the second kind) are presented. In Section 6, we give some remarks and observations on our results related to some probability distributions such as the binomial distribution and the Poisson distribution. In Section 7, we conclude our findings.

\section{New Families of the Combinatorial Numbers and Polynomials}

In this section, we define new families of the combinatorial numbers and polynomials by the following generating functions, respectively:

$$
\mathcal{G}(t, k ; \lambda)=2^{-k}(\lambda(1+\lambda t)-1)^{k}=\sum_{n=0}^{\infty} Y_{n}^{(-k)}(\lambda) \frac{t^{n}}{n !}
$$

and,

$$
\mathcal{G}(t, x, k ; \lambda)=\mathcal{G}(t, k ; \lambda)(1+\lambda t)^{x}=\sum_{n=0}^{\infty} Q_{n}(x ; \lambda, k) \frac{t^{n}}{n !}
$$

where $k \in \mathbb{N}$ and $\lambda$ is a real or complex number.

Combining Equations (21) and (22), we get:

$$
\sum_{n=0}^{\infty} Q_{n}(x ; \lambda, k) \frac{t^{n}}{n !}=\sum_{n=0}^{\infty} \sum_{j=0}^{n}\left(\begin{array}{l}
n \\
j
\end{array}\right) \lambda^{n-j} Y_{j}^{(-k)}(\lambda)(x)_{n-j} \frac{t^{n}}{n !}
$$

Comparing coefficient of $\frac{t^{n}}{n !}$ on both sides of the above equation, we arrive at the following theorem:

\section{Theorem 1.}

$$
Q_{n}(x ; \lambda, k)=\sum_{j=0}^{n}\left(\begin{array}{c}
n \\
j
\end{array}\right) \lambda^{n-j} Y_{j}^{(-k)}(\lambda)(x)_{n-j} .
$$


By the binomial theorem, we have:

$$
\sum_{n=0}^{\infty} Y_{n}^{(-k)}(\lambda) \frac{t^{n}}{n !}=2^{-k} \sum_{n=0}^{\infty}\left(\begin{array}{l}
k \\
n
\end{array}\right) \lambda^{2 n}(\lambda-1)^{k-n} t^{n}
$$

Comparing the coefficient of $t^{n}$ on both sides of the above equation, we arrive at the following theorem:

Theorem 2. Let $k$ and $n$ be nonnegative integers. Then:

$$
Y_{n}^{(-k)}(\lambda)=\left\{\begin{array}{cc}
2^{-k} n !\left(\begin{array}{c}
k \\
n
\end{array}\right) \lambda^{2 n}(\lambda-1)^{k-n} & \text { if } n \leq k \\
0 & \text { if } n>k
\end{array}\right.
$$

By Equation (25), a few values of the numbers $Y_{n}^{(-k)}(\lambda)$ are computed as follows:

$$
\begin{aligned}
Y_{0}^{(-k)}(\lambda) & =2^{-k}(\lambda-1)^{k} \\
Y_{1}^{(-k)}(\lambda) & =2^{-k}\left(\begin{array}{c}
k \\
1
\end{array}\right) \lambda^{2}(\lambda-1)^{k-1}, \\
Y_{2}^{(-k)}(\lambda) & =2^{-k} 2 !\left(\begin{array}{l}
k \\
2
\end{array}\right) \lambda^{4}(\lambda-1)^{k-2}, \\
& \vdots \\
Y_{j}^{(-k)}(\lambda) & =2^{-k} j !\left(\begin{array}{c}
k \\
j
\end{array}\right) \lambda^{2 j}(\lambda-1)^{k-j} \text { for } j \leq k, \\
& \vdots \\
Y_{k}^{(-k)}(\lambda) & =2^{-k} k ! \lambda^{2 k}, \\
Y_{j}^{(-k)}(\lambda) & =0 \text { for } j>k .
\end{aligned}
$$

By Equations (24) and (25), we also compute a few values of the polynomials $Q_{n}(x ; \lambda, k)$ as follows:

$$
\begin{aligned}
Q_{0}(x ; \lambda, k)= & 2^{-k}(\lambda-1)^{k}, \\
Q_{1}(x ; \lambda, k)= & 2^{-k}(\lambda-1)^{k} \lambda x+2^{-k} k \lambda^{2}(\lambda-1)^{k-1}, \\
Q_{2}(x ; \lambda, k)= & 2^{-k}(\lambda-1)^{k} \lambda^{2} x^{2}+\left(-2^{-k}(\lambda-1)^{k} \lambda^{2}+2^{-k+1} k \lambda^{3}(\lambda-1)^{k-1}\right) x \\
& +2^{-k} k(k-1) \lambda^{4}(\lambda-1)^{k-1} .
\end{aligned}
$$

By Equation (3), we arrive at a computation formula, for the numbers $Y_{n}^{(-k)}(\lambda)$, in terms of the Bernstein basis functions by the following corollary:

Corollary 1. Let $n$ and $k$ be nonnegative integers and $\lambda \in[0,1]$. Then,

$$
Y_{n}^{(-k)}(\lambda)=\left\{\begin{array}{cl}
2^{-k} n !(-1)^{k-n} \lambda^{n} B_{n}^{k}(\lambda) & \text { if } n \leq k \\
0 & \text { if } n>k .
\end{array}\right.
$$

Replacing $1+\lambda t$ by $e^{\log (1+\lambda t)}$ leads Equation (21) to be:

$$
\sum_{n=0}^{\infty} Y_{n}^{(-k)}(\lambda) \frac{t^{n}}{n !}=\frac{(-1)^{k}}{2^{k}}\left(-\lambda e^{\log (1+\lambda t)}+1\right)^{k}
$$


By combining Equation (17) with the above quation, we get:

$$
\sum_{n=0}^{\infty} Y_{n}^{(-k)}(\lambda) \frac{t^{n}}{n !}=\frac{(-1)^{k} k !}{2^{k}} \sum_{m=0}^{\infty} y_{1}(m, k ;-\lambda) \frac{(\log (1+\lambda t))^{m}}{m !}
$$

which follows from Equation (5) that:

$$
\sum_{n=0}^{\infty} Y_{n}^{(-k)}(\lambda) \frac{t^{n}}{n !}=\frac{(-1)^{k} k !}{2^{k}} \sum_{n=0}^{\infty} \sum_{m=0}^{n} \lambda^{n} y_{1}(m, k ;-\lambda) S_{1}(n, m) \frac{t^{n}}{n !}
$$

Therefore, by comparing coefficient of $\frac{t^{n}}{n !}$ on both sides of the above equation, we arrive at the following theorem:

Theorem 3.

$$
Y_{n}^{(-k)}(\lambda)=\frac{(-1)^{k} k !}{2^{k}} \sum_{m=0}^{n} \lambda^{n} y_{1}(m, k ;-\lambda) S_{1}(n, m)
$$

Combining Equations (25) with (30) yields the following corollary:

\section{Corollary 2.}

$$
\sum_{m=0}^{n} y_{1}(m, k ;-\lambda) S_{1}(n, m)=\left\{\begin{array}{clc}
\frac{(-1)^{k} \lambda^{n}(\lambda-1)^{k-n}}{(k-n) !} & \text { if } n \leq k \\
0 & \text { if } n>k
\end{array}\right.
$$

If we also combine Equations (26) with (30), then we have the following result:

Corollary 3. Let $n$ and $k$ nonnegative integer with $n \leq k$. Then,

$$
\sum_{m=0}^{n} y_{1}(m, k ;-\lambda) S_{1}(n, m)=(-1)^{n} \frac{n !}{k !} B_{n}^{k}(\lambda)
$$

On the other hand, since the following equality holds true (cf. [13]):

$$
S_{2}(n, k ; \lambda)=(-1)^{k} y_{1}(n, k ;-\lambda),
$$

Equation (31) leads the following corollary:

\section{Corollary 4.}

$$
\sum_{m=0}^{n} S_{2}(m, k ; \lambda) S_{1}(n, m)=\left\{\begin{array}{ccc}
\frac{\lambda^{n}(\lambda-1)^{k-n}}{(k-n) !} & \text { if } & n \leq k \\
0 & \text { if } & n>k
\end{array}\right.
$$

\section{Derivative Formulas and Recurrence Relations Arising from Differential Equations of Generating Functions}

In this section, by using differential equations involving the generating functions $\mathcal{G}(t, k ; \lambda)$ and $\mathcal{G}(t, x, k ; \lambda)$ and their partial derivatives with respect to the parameters $t, \lambda$, and $x$, we obtain some derivative formulas and recurrence relations for the numbers $Y_{n}^{(-k)}(\lambda)$ and the polynomials $Q_{n}(x ; \lambda, k)$.

Differentiating both sides of Equation (21) with respect to $\lambda$, we get the following partial derivative equation:

$$
\frac{\partial}{\partial \lambda}\{\mathcal{G}(t, k ; \lambda)\}=\frac{k}{2}(2 \lambda t+1) \mathcal{G}(t, k-1 ; \lambda)
$$


Also, if we differentiate both sides of Equation (21) with respect to $t$, then we get the following partial derivative equation:

$$
\frac{\partial}{\partial t}\{\mathcal{G}(t, k ; \lambda)\}=\frac{k \lambda^{2}}{2} \mathcal{G}(t, k-1 ; \lambda) .
$$

By combining Equation (35) with the RHS of Equation (21), we obtain:

$$
\sum_{n=0}^{\infty} \frac{d}{d \lambda}\left\{Y_{n}^{(-k)}(\lambda)\right\} \frac{t^{n}}{n !}=\frac{k}{2} \sum_{n=0}^{\infty}\left(2 n \lambda Y_{n-1}^{(-k+1)}(\lambda)+Y_{n}^{(-k+1)}(\lambda)\right) \frac{t^{n}}{n !} .
$$

Comparing the coefficients of $\frac{t^{n}}{n !}$ on both sides of the above equation, we arrive at the following theorem:

Theorem 4. Let $n \in \mathbb{N}$. Then, we have:

$$
\frac{d}{d \lambda}\left\{Y_{n}^{(-k)}(\lambda)\right\}=\frac{k}{2}\left(2 n \lambda Y_{n-1}^{(-k+1)}(\lambda)+Y_{n}^{(-k+1)}(\lambda)\right) .
$$

By combining Equation (36) with the RHS of Equation (21), we get:

$$
\frac{\partial}{\partial t} \sum_{n=0}^{\infty} Y_{n}^{(-k)}(\lambda) \frac{t^{n}}{n !}=\frac{k \lambda^{2}}{2} \sum_{n=0}^{\infty} Y_{n}^{(-k+1)}(\lambda) \frac{t^{n}}{n !}
$$

which, by comparing the coefficients of $\frac{t^{n}}{n !}$ on both sides of the above equation, yields the following theorem:

Theorem 5. Let $n \in \mathbb{N}_{0}$. Then, we have:

$$
Y_{n+1}^{(-k)}(\lambda)=\frac{k \lambda^{2}}{2} Y_{n}^{(-k+1)}(\lambda)
$$

Differentiating both sides of Equation (22) with respect to $\lambda$, we get the following partial derivative equation:

$$
\frac{\partial}{\partial \lambda}\{\mathcal{G}(t, x, k ; \lambda)\}=\frac{k}{2}(2 \lambda t+1) \mathcal{G}(t, x, k-1 ; \lambda)+x t \mathcal{G}(t, x-1, k ; \lambda) .
$$

Furthermore, if we differentiate both sides of the Equation (22) with respect to $t$, then we also get the following partial derivative equation:

$$
\frac{\partial}{\partial t}\{\mathcal{G}(t, x, k ; \lambda)\}=\frac{k \lambda^{2}}{2} \mathcal{G}(t, x, k-1 ; \lambda)+x \lambda \mathcal{G}(t, x-1, k ; \lambda) .
$$

Additionally, when we differentiate both sides of Equation (22) with respect to $x$, we also get the following partial derivative equation:

$$
\frac{\partial}{\partial x}\{\mathcal{G}(t, x, k ; \lambda)\}=\log (1+\lambda t) \mathcal{G}(t, x, k ; \lambda) .
$$

By combining Equation (41) with the RHS of Equation (22), we get:

$$
\sum_{n=0}^{\infty} \frac{\partial}{\partial \lambda}\left\{Q_{n}(x ; \lambda, k)\right\} \frac{t^{n}}{n !}=\frac{k}{2}(2 \lambda t+1) \sum_{n=0}^{\infty} Q_{n}(x ; \lambda, k-1) \frac{t^{n}}{n !}+x t \sum_{n=0}^{\infty} Q_{n}(x-1 ; \lambda, k) \frac{t^{n}}{n !}
$$


which yields:

$$
\begin{aligned}
\sum_{n=0}^{\infty} \frac{\partial}{\partial \lambda}\left\{Q_{n}(x ; \lambda, k)\right\} \frac{t^{n}}{n !}= & \frac{k}{2} \sum_{n=0}^{\infty}\left(2 n \lambda Q_{n-1}(x ; \lambda, k-1)+Q_{n}(x ; \lambda, k-1)\right) \frac{t^{n}}{n !} \\
& +x \sum_{n=0}^{\infty} n Q_{n-1}(x-1 ; \lambda, k) \frac{t^{n}}{n !} .
\end{aligned}
$$

Comparing the coefficients of $\frac{t^{n}}{n !}$ on both sides of the above equation, we arrive at the following theorem:

Theorem 6. Let $n \in \mathbb{N}$. Then, we have:

$$
\frac{\partial}{\partial \lambda}\left\{Q_{n}(x ; \lambda, k)\right\}=k n \lambda Q_{n-1}(x ; \lambda, k-1)+\frac{k}{2} Q_{n}(x ; \lambda, k-1)+x n Q_{n-1}(x-1 ; \lambda, k) .
$$

By combining Equation (42) with the RHS of Equation (22), we get:

$$
\frac{\partial}{\partial t} \sum_{n=0}^{\infty} Q_{n}(x ; \lambda, k) \frac{t^{n}}{n !}=\frac{k \lambda^{2}}{2} \sum_{n=0}^{\infty} Q_{n}(x ; \lambda, k-1) \frac{t^{n}}{n !}+x \lambda \sum_{n=0}^{\infty} Q_{n}(x-1 ; \lambda, k) \frac{t^{n}}{n !}
$$

which, by comparing the coefficients of $\frac{t^{n}}{n !}$ on both sides of the above equation, yields the following theorem:

Theorem 7. Let $n \in \mathbb{N}_{0}$. Then, we have:

$$
Q_{n+1}(x ; \lambda, k)=\frac{k \lambda^{2}}{2} Q_{n}(x ; \lambda, k-1)+x \lambda Q_{n}(x-1 ; \lambda, k) .
$$

By combining Equation (43) with the RHS of Equation (22) and the Taylor series of the function $\log (1+\lambda t)$, we get:

$$
\sum_{n=0}^{\infty} \frac{\partial}{\partial x}\left\{Q_{n}(x ; \lambda, k)\right\} \frac{t^{n}}{n !}=\sum_{n=1}^{\infty}(-1)^{n-1} \frac{\lambda^{n} t^{n}}{n} \sum_{n=0}^{\infty} Q_{n}(x ; \lambda, k) \frac{t^{n}}{n !} .
$$

Applying the Cauchy product rule to the above equation yields:

$$
\sum_{n=0}^{\infty} \frac{\partial}{\partial x}\left\{Q_{n}(x ; \lambda, k)\right\} \frac{t^{n}}{n !}=t \sum_{n=0}^{\infty}\left(\sum_{j=0}^{n}(-1)^{j}\left(\begin{array}{c}
n \\
j
\end{array}\right) \frac{j ! \lambda^{j+1}}{j+1} Q_{n-j}(x ; \lambda, k)\right) \frac{t^{n}}{n !} .
$$

Comparing the coefficients of $\frac{t^{n}}{n !}$ on both sides of the above equation, we arrive at the following theorem:

Theorem 8. Let $n \in \mathbb{N}$. Then, we have:

$$
\frac{\partial}{\partial x}\left\{Q_{n}(x ; \lambda, k)\right\}=n \sum_{j=0}^{n-1}(-1)^{j}\left(\begin{array}{c}
n-1 \\
j
\end{array}\right) \frac{j ! \lambda^{j+1}}{j+1} Q_{n-1-j}(x ; \lambda, k) .
$$

Remark 1. Substituting Equation (16) into Equation (46) yields the following formula including Daehee numbers:

$$
\frac{\partial}{\partial x}\left\{Q_{n}(x ; \lambda, k)\right\}=n \sum_{j=0}^{n-1}\left(\begin{array}{c}
n-1 \\
j
\end{array}\right) \lambda^{j+1} D_{j} Q_{n-1-j}(x ; \lambda, k) .
$$


By Equation (15), another form of the partial differential Equation (43) is given by:

$$
\frac{\partial}{\partial x}\{\mathcal{G}(t, x, k ; \lambda)\}=\lambda t \mathcal{G}(t, k ; \lambda) F_{D}(x, \lambda t) .
$$

By combining Equation (48) with the RHS of the Equations (15) and (22), we get:

$$
\sum_{n=0}^{\infty} \frac{\partial}{\partial x}\left\{Q_{n}(x ; \lambda, k)\right\} \frac{t^{n}}{n !}=\lambda t \sum_{n=0}^{\infty} Y_{n}^{(-k)}(\lambda) \frac{t^{n}}{n !} \sum_{n=0}^{\infty} \lambda^{n} D_{n}(x) \frac{t^{n}}{n !}
$$

Applying the Cauchy product rule to the above equation yields:

$$
\sum_{n=0}^{\infty} \frac{\partial}{\partial x}\left\{Q_{n}(x ; \lambda, k)\right\} \frac{t^{n}}{n !}=\lambda t \sum_{n=0}^{\infty}\left(\sum_{j=0}^{n} \lambda^{j}\left(\begin{array}{c}
n \\
j
\end{array}\right) Y_{n-j}^{(-k)}(\lambda) D_{j}(x)\right) \frac{t^{n}}{n !} .
$$

Comparing the coefficients of $\frac{t^{n}}{n !}$ on both sides of the above equation, we arrive at the following theorem:

\section{Theorem 9.}

$$
\frac{\partial}{\partial x}\left\{Q_{n}(x ; \lambda, k)\right\}=n \sum_{j=0}^{n-1} \lambda^{j+1}\left(\begin{array}{c}
n-1 \\
j
\end{array}\right) Y_{n-j}^{(-k)}(\lambda) D_{j}(x)
$$

\section{Some Identities and Relations Derived from Functional Equations of Generating Functions}

In this section, by using functional equations of the aforementioned generating functions, we derive some formulas and combinatorial sums including binomials coefficients, falling factorial, the Stirling numbers, the Bell polynomials (i.e., exponential polynomials), the Poisson-Charlier polynomials, combinatorial numbers and polynomials, and the Bersntein basis functions.

Now, we set the following functional equation:

$$
F_{p c}(t, x ; a)=\mathcal{F}\left(t, x, k ; \frac{1}{a}\right) \mathcal{F}\left(t,-k ; \frac{1}{a}\right) e^{-t} .
$$

Combining the above equation with the Equations (1), (11), and (12), we get:

$$
\sum_{n=0}^{\infty} C_{n}(x ; a) \frac{t^{n}}{n !}=\sum_{n=0}^{\infty} Y_{n}^{(k)}\left(x ; \frac{1}{a}\right) \frac{t^{n}}{n !} \sum_{n=0}^{\infty} Y_{n}^{(-k)}\left(\frac{1}{a}\right) \frac{t^{n}}{n !} \sum_{n=0}^{\infty}(-1)^{n} \frac{t^{n}}{n !} .
$$

Applying the Cauchy product rule to the above equation yields:

$$
\sum_{n=0}^{\infty} C_{n}(x ; a) \frac{t^{n}}{n !}=\sum_{n=0}^{\infty}\left(\sum_{l=0}^{n} \sum_{j=0}^{l}(-1)^{n-l}\left(\begin{array}{l}
n \\
l
\end{array}\right)\left(\begin{array}{l}
l \\
j
\end{array}\right) Y_{j}^{(k)}\left(x ; \frac{1}{a}\right) Y_{l-j}^{(-k)}\left(\frac{1}{a}\right)\right) \frac{t^{n}}{n !} .
$$

Therefore, by comparing the coefficient of $\frac{t^{n}}{n !}$ on both sides of the above equation, we arrive at the following theorem:

Theorem 10.

$$
C_{n}(x ; a)=\sum_{l=0}^{n} \sum_{j=0}^{l}(-1)^{n-l}\left(\begin{array}{l}
n \\
l
\end{array}\right)\left(\begin{array}{l}
l \\
j
\end{array}\right) Y_{j}^{(k)}\left(x ; \frac{1}{a}\right) Y_{l-j}^{(-k)}\left(\frac{1}{a}\right) .
$$

Moreover, we also set the following functional equation:

$$
F_{B}(t,-x ; k) F_{p c}(t, x ; a)=\frac{(-1)^{k}(x t)^{k}}{k !} e^{x t}\left(\frac{t}{a}+1\right)^{x} .
$$


Combining the above equation with the Equations (1) and (4) yields:

$$
\sum_{n=0}^{\infty} B_{k}^{n}(-x) \frac{t^{n}}{n !} \sum_{n=0}^{\infty} C_{n}(x ; a) \frac{t^{n}}{n !}=\frac{(-1)^{k}(x t)^{k}}{k !} \sum_{n=0}^{\infty} \frac{(x t)^{n}}{n !} \sum_{n=0}^{\infty}\left(\begin{array}{l}
x \\
n
\end{array}\right) \frac{t^{n}}{a^{n}} .
$$

Applying the Cauchy product rule to the above equation yields:

$$
\sum_{n=0}^{\infty}\left(\sum_{j=0}^{n}\left(\begin{array}{c}
n \\
j
\end{array}\right) B_{k}^{j}(-x) C_{n-j}(x ; a)\right) \frac{t^{n}}{n !}=\frac{(-1)^{k} x^{k}}{k !} \sum_{n=0}^{\infty}\left((n)_{k} \sum_{j=0}^{n-k}\left(\begin{array}{c}
n-k \\
j
\end{array}\right) \frac{x^{n-k-j}(x)_{j}}{a^{j}}\right) \frac{t^{n}}{n !} .
$$

Therefore, by comparing the coefficient of $\frac{t^{n}}{n !}$ on both sides of the above equation, we arrive at the following theorem:

Theorem 11.

$$
\sum_{j=0}^{n}\left(\begin{array}{c}
n \\
j
\end{array}\right) B_{k}^{j}(-x) C_{n-j}(x ; a)=\frac{(-1)^{k}(n)_{k}}{k !} \sum_{j=0}^{n-k}\left(\begin{array}{c}
n-k \\
j
\end{array}\right) \frac{x^{n-j}(x)_{j}}{a^{j}}
$$

Additionaly, we also have the following functional equation:

$$
\frac{(x t)^{k}}{k !} F_{p c}(-t, x ; a) e^{-x t}=F_{B}(t, x ; k)\left(-\frac{t}{a}+1\right)^{x} .
$$

Combining the above equation with Equations (1) and (4) yields:

$$
\frac{(x t)^{k}}{k !} \sum_{n=0}^{\infty}(-1)^{n} C_{n}(x ; a) \frac{t^{n}}{n !} \sum_{n=0}^{\infty}(-x)^{n} \frac{t^{n}}{n !}=\sum_{n=0}^{\infty} B_{k}^{n}(x) \frac{t^{n}}{n !} \sum_{n=0}^{\infty}(-1)^{n}\left(\begin{array}{l}
x \\
n
\end{array}\right) \frac{t^{n}}{a^{n}} .
$$

Applying the Cauchy product rule to the above equation yields:

$$
\sum_{n=0}^{\infty}\left(\frac{(-1)^{n-k}(n)_{k}}{k !} \sum_{j=0}^{n-k}\left(\begin{array}{c}
n-k \\
j
\end{array}\right) x^{n-j} C_{j}(x ; a)\right) \frac{t^{n}}{n !}=\sum_{n=0}^{\infty}\left(\sum_{j=0}^{n}(-1)^{j}\left(\begin{array}{c}
n \\
j
\end{array}\right) \frac{(x)_{j} B_{k}^{n-j}(x)}{a^{j}}\right) \frac{t^{n}}{n !} .
$$

Therefore, by comparing coefficient of $\frac{t^{n}}{n !}$, we arrive at the following theorem:

\section{Theorem 12.}

$$
\frac{(-1)^{n-k}(n)_{k}}{k !} \sum_{j=0}^{n-k}\left(\begin{array}{c}
n-k \\
j
\end{array}\right) x^{n-j} C_{j}(x ; a)=\sum_{j=0}^{n}(-1)^{j}\left(\begin{array}{c}
n \\
j
\end{array}\right) \frac{(x)_{j} B_{k}^{n-j}(x)}{a^{j}} .
$$

By substituting $t \rightarrow a\left(e^{t}-1\right)$ into Equation (1), we also get the following functional equation:

$$
F_{p c}\left(a\left(e^{t}-1\right), x ; a\right)=e^{t x} F_{\text {Bell }}(t,-a) .
$$

By Equations (1) and (10), we thus get:

$$
\sum_{n=0}^{\infty} a^{n} C_{n}(x ; a) \frac{\left(e^{t}-1\right)^{n}}{n !}=\sum_{n=0}^{\infty} x^{n} \frac{t^{n}}{n !} \sum_{n=0}^{\infty} B l_{n}(-a) \frac{t^{n}}{n !} .
$$


Applying the Cauchy product rule to the above equation and combining Equation (8) with the final equation yields:

$$
\sum_{m=0}^{\infty} \sum_{n=0}^{m} a^{n} C_{n}(x ; a) S_{2}(m, n) \frac{t^{m}}{m !}=\sum_{m=0}^{\infty}\left(\sum_{j=0}^{m}\left(\begin{array}{c}
m \\
j
\end{array}\right) x^{m-j} B l_{j}(-a)\right) \frac{t^{m}}{m !} .
$$

Therefore, by comparing the coefficient of $\frac{t^{m}}{m !}$, we arrive at the following theorem:

\section{Theorem 13.}

$$
\sum_{n=0}^{m} a^{n} C_{n}(x ; a) S_{2}(m, n)=\sum_{j=0}^{m}\left(\begin{array}{c}
m \\
j
\end{array}\right) x^{m-j} B l_{j}(-a)
$$

\section{Some Identities and Relations Arising from the $p$-adic Integrals and Riemann Integral}

In this section, by applying the $p$-adic integrals and Riemann integral to some of our results, we derive some combinatorial sums including the binomial coefficients, falling factorial, the Bernoulli numbers, the Euler numbers, the Stirling numbers, the Bell polynomials (i.e., exponential polynomials), and the Cauchy numbers (or the Bernoulli numbers of the second kind).

Let $\mathbb{Z}_{p}$ denote a set of $p$-adic integers. Let $f(x)$ be a uniformly differentiable function on $\mathbb{Z}_{p}$. The Volkenborn integral (or $p$-adic bosonic integral) of the function $f(x)$ is given by:

$$
\int_{\mathbb{Z}_{p}} f(x) d \mu_{1}(x)=\lim _{N \rightarrow \infty} \frac{1}{p^{N}} \sum_{x=0}^{p^{N}-1} f(x),
$$

where,

$$
\mu_{1}(x)=\mu_{1}\left(x+p^{N} \mathbb{Z}_{p}\right)=\frac{1}{p^{N}}
$$

(cf. [17]; see also [11,12]).

It is known that the bosonic $p$-adic integral of the function $f(x)=x^{n}$ gives the Bernoulli numbers as follows $(c f .[11,17])$ :

$$
B_{n}=\int_{\mathbb{Z}_{p}} x^{n} d \mu_{1}(x)
$$

where $B_{n}$ denotes the Bernoulli numbers of the first kind defined by means of the following generating function:

$$
\frac{t}{e^{t}-1}=\sum_{n=0}^{\infty} B_{n} \frac{t^{n}}{n !}, \quad(t<|2 \pi|)
$$

which arise in not only analytic number theory, but also other related areas (cf. [5-31]).

The fermionic $p$-adic integral of the function $f(x)$ is given by (cf. [12]):

$$
\int_{\mathbb{Z}_{p}} f(x) d \mu_{-1}(x)=\lim _{N \rightarrow \infty} \sum_{x=0}^{p^{N}-1}(-1)^{x} f(x)
$$

where $p \neq 2$ and,

$$
\mu_{-1}(x)=\mu_{-1}\left(x+p^{N} \mathbb{Z}_{p}\right)=\frac{(-1)^{x}}{p^{N}}
$$

(cf. $[10,12])$. 
The fermionic $p$-adic integral of the function $f(x)=x^{n}$ gives the Euler numbers as follows (cf. [11]):

$$
E_{n}=\int_{\mathbb{Z}_{p}} x^{n} d \mu_{-1}(x),
$$

where $E_{n}$ denotes the Euler numbers of the first kind defined by means of the following generating function:

$$
\frac{2}{e^{t}+1}=\sum_{n=0}^{\infty} E_{n} \frac{t^{n}}{n !}, \quad(t<|\pi|)
$$

(cf. [5-30]).

It is known that the following $p$-adic bosonic and fermionic integral representations for the Poisson-Charlier polynomials hold true (see [24] (Equations (33) and (35), pp. 944-945)):

$$
\int_{\mathbb{Z}_{p}} C_{n}(x ; a) d \mu_{1}(x)=\sum_{k=0}^{n}(-1)^{n}\left(\begin{array}{l}
n \\
k
\end{array}\right) \frac{k !}{(k+1) a^{k}}
$$

and,

$$
\int_{\mathbb{Z}_{p}} C_{n}(x ; a) d \mu_{-1}(x)=\sum_{k=0}^{n}(-1)^{n}\left(\begin{array}{l}
n \\
k
\end{array}\right) \frac{k !}{(2 a)^{k}} .
$$

By applying the bosonic $p$-adic integral to Equation (58) and combining the final equation with Equations (60) and (65), we arrive at the following theorem:

Theorem 14.

$$
\sum_{n=0}^{m} \sum_{k=0}^{n}(-1)^{n} \frac{(n)_{k} a^{n-k} S_{2}(m, n)}{k+1}=\sum_{j=0}^{m}\left(\begin{array}{c}
m \\
j
\end{array}\right) B_{m-j} B l_{j}(-a) .
$$

By applying the fermionic $p$-adic integral to Equation (58) and combining the final equation with Equations (63) and (66), we also arrive at the following theorem:

Theorem 15.

$$
\sum_{n=0}^{m} \sum_{k=0}^{n}(-1)^{n} \frac{(n)_{k} a^{n-k} S_{2}(m, n)}{2^{k}}=\sum_{j=0}^{m}\left(\begin{array}{c}
m \\
j
\end{array}\right) E_{m-j} B l_{j}(-a) .
$$

Moreover, by integrating Equation (58) with respect to $x$ from 0 to 1, we have:

$$
\sum_{n=0}^{m} a^{n} S_{2}(m, n) \int_{0}^{1} C_{n}(x ; a) d x=\sum_{j=0}^{m}\left(\begin{array}{c}
m \\
j
\end{array}\right) B l_{j}(-a) \int_{0}^{1} x^{m-j} d x .
$$

On the other hand, by integrating Equation (2) with respect to $x$ from 0 to 1 , we also have:

$$
\int_{0}^{1} C_{n}(x ; a) d x=\sum_{j=0}^{n}(-1)^{n-j}\left(\begin{array}{c}
n \\
j
\end{array}\right) \frac{1}{a^{j}} \int_{0}^{1}(x)_{j} d x,
$$

By making use of the following definition of the well-known Cauchy numbers (or the Bernoulli numbers of the second kind) $b_{n}(0)(c f .[4])$ :

$$
b_{n}(0)=\int_{0}^{1}(x)_{n} d x
$$


Equation (70) yields:

$$
\int_{0}^{1} C_{n}(x ; a) d x=\sum_{j=0}^{n}(-1)^{n-j}\left(\begin{array}{c}
n \\
j
\end{array}\right) \frac{b_{j}(0)}{a^{j}} .
$$

Combining the above equation with Equation (69), we arrive at the following theorem:

\section{Theorem 16 .}

$$
\sum_{n=0}^{m} \sum_{j=0}^{n}(-1)^{n-j}\left(\begin{array}{c}
n \\
j
\end{array}\right) a^{n-j} S_{2}(m, n) b_{j}(0)=\sum_{j=0}^{m}\left(\begin{array}{c}
m \\
j
\end{array}\right) \frac{B l_{j}(-a)}{m-j+1}
$$

\section{Applications in the Probability Distribution Function}

In this section, we investigate some applications of the numbers $Y_{n}^{(-k)}(\lambda)$. Assume that $0<p \leq 1$ and $n=0,1,2, \ldots, k$. We set the following discrete probability distribution:

$$
f(p ; k, n)=\frac{(-1)^{k-n} 2^{k}}{n ! p^{n}} Y_{n}^{(-k)}(p)
$$

where $p$ is a probability of success, $k$ is number of trials, $n$ is number of successes in $k$ trials, and $n=0,1,2, \ldots, k$. Therefore, $f(p ; k, n)$ is binomially distributed with parameters $(k, p)$.

Properties of Discrete Probability Distribution $f(p ; k, n)$

Here, we give some properties of discrete probability distribution $f(p ; k, n)$. We examine the properties of the probability distribution $f(p ; k, n)$ with a random variable with parameters $k, n$, and $p$ as follows:

For all $k, n, p$ with $0 \leq n \leq k$ and $0<p \leq 1,0 \leq f(p ; k, n) \leq 1$. That is $f(p ; k, n) \geq 0$.

The probability distribution function $f(p ; k, n)$ satisfies that:

$$
\sum_{n=0}^{\infty} f(p ; k, n)=1
$$

Computing the distribution function $f(p ; k, n)$. Suppose that $X$ is a binomial with parameters $(k, p)$. To computing its distribution function:

$$
P(X \leq j)=\sum_{n=0}^{j} f(p ; k, n),
$$

where $j=0,1, \ldots, k$.

In order to compute its expected value and variance for random variable with parameters $k$ and $p$ :

$$
E\left[X^{v}\right]=\sum_{n=0}^{k} n^{v} f(p ; k, n)
$$

Observe that the probability distribution function $f(p ; k, n)$ is a modification of the binomial probability distribution function with parameters $(k, p)$. Substituting $v=1$ into Equation (75), $E[X]=$ $k p$. Substituting $v=2$ into Equation (75), variance $E\left[X^{2}\right]-(E[X])^{2}=k p(1-p)$.

If we take $k \rightarrow \infty$, then the distribution $f(p ; k, n)$ goes to the Poisson distribution. On the other hand the Poisson-Charlier polynomials are orthogonal with respect to the Poisson distribution (cf. $[18,24])$. 


\section{Conclusions}

Applications of generating functions are used in many areas, and we used them to study new families of combinatorial numbers and polynomials. We then studied properties of these new families, which yielded a handful of new identities and relations. Namely, these identities were related to numerous special numbers, special polynomials, and special functions such as the Bersntein basis functions, the Stirling numbers, the Bell polynomials (or exponential polynomials), the Poisson-Charlier polynomials, and the probability distribution functions. Furthermore, we should note that newly defined combinatorial numbers in this paper gave a different approach to the binomial (or Newton) distribution and the Poisson distribution, as well as combinatorial sums including the Bernoulli numbers, the Euler numbers, the Cauchy numbers (or the Bernoulli numbers of the second kind), and combinatorial numbers. This is why the results of this paper have the potential to be used in numerous areas such as mathematics, probability, physics, and in other associated areas.

Author Contributions: Investigation, I.K., B.S., Y.S.; wirting-original draft, I.K., B.S., Y.S.; writing-review and editing, I.K., B.S., Y.S.

Funding: This research received no external funding.

Acknowledgments: This paper is dedicated to Hari Mohan Srivastava on the occasion of his 80th Birthday. Yilmaz Simsek was supported by the Scientific Research Project Administration of Akdeniz University.

Conflicts of Interest: The authors declare no conflict of interest.

\section{References}

1. Acikgoz, M.; Araci, S. On generating function of the Bernstein polynomials. Proc. Int. Conf. Numer. Anal. Appl. Math. Am. Inst. Phys. Conf. Proc. 2010, CP1281, 1141-1143.

2. Bona, M. Introduction to Enumerative Combinatorics; The McGraw-Hill Companies Inc.: New York, NY, USA, 2007.

3. Charalambides, C.A. Enumerative Combinatorics; Chapman and Hall (CRC Press Company): London, UK; New York, NY, USA, 2002.

4. Comtet, L. Advanced Combinatorics: The Art of Finite and Infinite Expansions; D. Reidel Publishing Company: Dordrecht, The Netherlands; Boston, MA, USA, 1974.

5. Djordjevic, G.B.; Milovanović, G.V. Special Classes of Polynomials; Faculty of Technology, University of Nis: Leskovac, Serbia, 2014.

6. Golombek, R. Aufgabe 1088. El. Math. 1994, 49, 126-127.

7. Jordan, C. Calculus of Finite Differences, 2nd ed.; Chelsea Publishing Company: New York, NY, USA, 1950.

8. Kim, D.S.; Kim, T. Daehee numbers and polynomials. Appl. Math. Sci. (Ruse) 2013, 7, 5969-5976. [CrossRef]

9. Kim, D.S.; Kim, T.; Seo, J. A note on Changhee numbers and polynomials. Adv. Stud. Theor. Phys. 2013, 7, 993-1003. [CrossRef]

10. Kim, M.-S. On Euler numbers, polynomials and related p-adic integrals. J. Number Theory 2009, 129, 2166-2179. [CrossRef]

11. Kim, T. q-Volkenborn integration. Russ. J. Math. Phys. 2002, 19, 288-299.

12. Kim, T. $q$-Euler numbers and polynomials associated with $p$-adic $q$-integral and basic $q$-zeta function. Trend Math. Inf. Cent. Math. Sci. 2006, 9, 7-12.

13. Kucukoglu, I.; Simsek, Y. Observations on identities and relations for interpolation functions and special numbers. Adv. Stud. Contemp. Math. 2018, 28, 41-56.

14. Kucukoglu, I.; Simsek, B.; Simsek, Y. An approach to negative hypergeometric distribution by generating function for special numbers and polynomials. Turk. J. Math. 2019, 43, 2337-2353.

15. Lorentz, G.G. Bernstein Polynomials; Chelsea Publishing Company: New York, NY, USA, 1986.

16. Ozmen, N.; Erkus-Duman, E. On the Poisson-Charlier polynomials. Serdica Math. J. 2015, 41, 457-470.

17. Schikhof, W.H. Ultrametric Calculus: An Introduction to p-Adic Analysis; Cambridge Studies in Advanced Mathematics 4; Cambridge University Press: Cambridge, UK, 1984.

18. Roman, S. The Umbral Calculus; Dover Publishing Inc.: New York, NY, USA, 2005.

19. Ross, S.M. A First Course in Probability, 8th ed.; Pearson Education, Inc.: London, UK, 2010. 
20. Simsek, Y. Functional equations from generating functions: A novel approach to deriving identities for the Bernstein basis functions. Fixed Point Theory Appl. 2013, 2013, 1-13. [CrossRef]

21. Simsek, Y. Generating functions for generalized Stirling type numbers, array type polynomials, Eulerian type polynomials and their applications. Fixed Point Theory Appl. 2013, 2013, 343-355. [CrossRef]

22. Simsek, Y. New families of special numbers for computing negative order Euler numbers and related numbers and polynomials. Appl. Anal. Discr. Math. 2018, 12,1-35. [CrossRef]

23. Simsek, Y. Construction of Some New Families of Apostol-type Numbers and Polynomials via Dirichlet Character and $p$-adic $q$-integrals. Turk. J. Math. 2018, 42, 557-577. [CrossRef]

24. Simsek, Y. Formulas for Poisson-Charlier, Hermite, Milne-Thomson and other type polynomials by their generating functions and p-adic integral approach. RACSAM Rev. R. Acad. A 2019, 113, 931-948. [CrossRef]

25. Simsek, Y. Generating functions for finite sums involving higher powers of binomial coefficients: Analysis of hypergeometric functions including new families of polynomials and numbers. J. Math. Anal. Appl. 2019, 477, 1328-1352. [CrossRef]

26. Simsek, Y.; Acikgoz, M. A new generating function of ( $q$-) Bernstein-type polynomials and their interpolation function. Abstr. Appl. Anal. 2010, 769095. [CrossRef]

27. Srivastava, H.M. Some formulas for the Bernoulli and Euler polynomials at rational arguments. Math. Proc. Camb. Philos. Soc. 2000, 129, 77-84. [CrossRef]

28. Srivastava, H.M. Some generalizations and basic (or $q$-) extensions of the Bernoulli, Euler and Genocchi polynomials. Appl. Math. Inf. Sci. 2011, 5, 390-444.

29. Srivastava, H.M.; Choi, J. Series Associated with the Zeta and Related Functions; Kluwer Acedemic Publishers: Dordrecht, The Netherlands; Boston, MA, USA; London, UK, 2001.

30. Srivastava, H.M.; Choi, J. Zeta and q-Zeta Functions and Associated Series and Integrals; Elsevier Science Publishers: Amsterdam, The Netherlands; London, UK; New York, NY, USA, 2012.

31. $\mathrm{Xu}, \mathrm{A}$. On an open problem of Simsek concerning the computation of a family special numbers. Appl. Anal. Discret. Math. 2019, 13, 61-72. [CrossRef]

(C) 2019 by the authors. Licensee MDPI, Basel, Switzerland. This article is an open access article distributed under the terms and conditions of the Creative Commons Attribution (CC BY) license (http:/ / creativecommons.org/licenses/by/4.0/). 